\title{
SCALABLE DETECTION OF PERCEPTUAL WATERMARKS IN JPEG2000 IMAGES
}

\author{
D. Simitopoulos, N. V. Boulgouris, A. Leontaris and M. G. Strintzis \\ Information Processing Laboratory \\ Department of Electrical and Computer Engineering \\ Aristotle University of Thessaloniki \\ Thessaloniki 540 06, Greece \\ and \\ Informatics and Telematics Institute \\ 1st Km Thermi-Panorama Road \\ 57001 (PO Box 361) \\ Thermi-Thessaloniki, Greece \\ dsim@iti.gr
}

Abstract In this paper, a novel watermarking technique for embedding information bits in JPEG2000 images is presented. The proposed scheme embeds the watermark in wavelet coefficients and is compatible with the line-based discrete wavelet transform that is used by JPEG2000. Further, the proposed method is coupled with wavelet-domain perceptual analysis to ensure that the watermark is invisible. The information bits carried by the watermark signal are protected using error correction codes. Watermark detection and information bit extraction is performed without the need of the original image. Experimental evaluation shows the reliability of the proposed watermarking method when used with the recently finalized JPEG2000 standard.

Keywords: Watermarking, JPEG2000, Perceptual analysis.

\section{INTRODUCTION}

The dominance of digital media in the storage, transmission and distribution of information is undeniable. The capability of making replica of digital media from unauthorized persons has rendered the need for their protection imperative. Still and moving images are the main form of digital media that has to be protected. Many methods have been proposed, based on image domain or transform domain processing. DCTdomain watermarking in particular has attracted much interest due to 
the fact that the current JPEG and MPEG-1/2 standards use DCT for coding information. The advent of the new image coding standard JPEG2000 offers a wide variety of new features in comparison to its predecessor JPEG. The deployment of the wavelet transform, instead of DCT, has intensified research on wavelet-domain methods for the efficient watermarking of images [Su et al., 2000].

Effective embedding of watermarks in images is dependent upon two contradicting factors: robustness and perceptual invisibility. Modern coders like JPEG2000 and SPIHT, provide even more challenges in designing an effective and robust watermarking algorithm. The aforementioned embedded coders produce fully scalable compressed image representations that gradually improve the decompressed image's quality as the transmitted data increases. Additionally, the capability of the new standard to deliver excellent image quality even at very low rates necessitates the investigation of watermarking schemes that are robust to quantization performed by JPEG2000. Thus, watermarks should be designed so that they remain statistically detectable even if only a small portion of the embedded bitstream is decoded. This paper proposes a data embedding scheme for JPEG2000 and evaluates its robustness against quantization.

The paper is organized as follows. Section 2 describes the proposed watermark embedding scheme. The watermark retrieval and error correction algorithm is presented in Section 3. In Section 4 experimental results are presented and finally, in Section 5, conclusions are drawn.

\section{PERCEPTUAL WATERMARK EMBEDDING}

The proposed watermark embedding scheme in its entirety is depicted in Figure 1. The embedding of information is performed in the discrete wavelet transform domain. The original image is decomposed in a four level multiresolution representation using the popular biorthogonal 9/7 filter bank [Antonini et al., 1992] which is the default filter bank for lossy compression in JPEG2000. The watermark is embedded in the HL, LH and $\mathrm{HH}$ bands of each resolution level. The watermark coefficients are drawn from a zero-mean, unit-variance gaussian distribution. Gaussian distributed samples are produced using an integer (32 bit) random number generator that is seeded with the result of a hash process. This process consists of the MD5 algorithm that, given a meaningful message (copyright information, owner ID) produces a 128 bit key, followed by another hash function that is fed with the 128 bit key as input and produces a 32 bit integer suitable for seeding. 


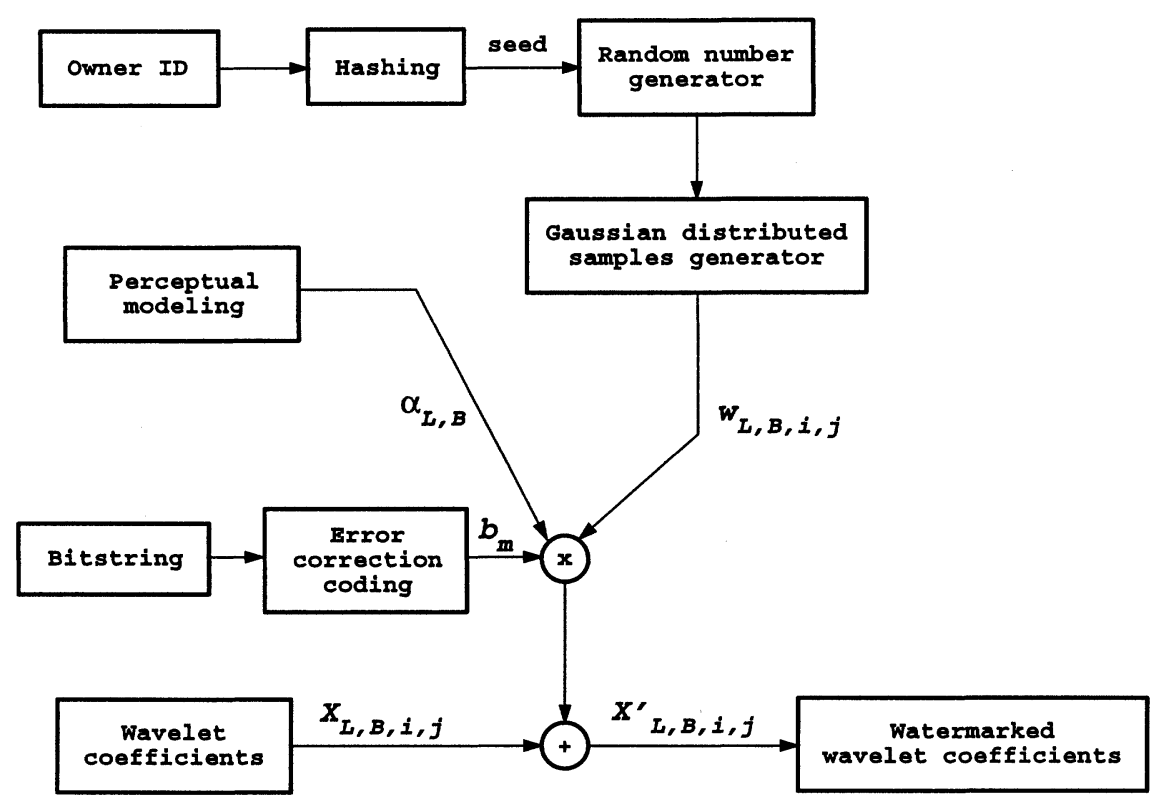

Figure 1 Embedding scheme.

Perceptual analysis is used in order to adapt the watermark to the host image. Watson et. al. [Watson et al., 1996] calculated the maximum quantizers $Q_{L, B}$, that, when applied to each band $B$ of each level $L$ of a DWT decomposed image, will not cause any perceptible degradation of the quality of the raw image. The values of the quantizers depend on the distance $d$ of the viewer in $\mathrm{cm}$, and the display resolution $p$ in pixels $/ \mathrm{cm}$, from which the display visual resolution $r$ in pixels/degree of visual angle is calculated as follows

$$
r=d p \frac{\pi}{180}
$$

Specifically, for a $48.7 \mathrm{~cm}$ viewing distance using a monitor with 37.65 pixels/cm display resolution the display visual resolution is valued 32 pixels/degree of visual angle. The corresponding $Q_{L, B}$ quantizers [Watson et al., 1996] are tabulated in Table 1. The level enumeration and the band labeling conventions are shown in Figure 2.

Therefore, each DWT coefficient may be altered by $a_{L, B}=Q_{L, B} / 2$ having an imperceptible effect on the image quality. For this reason, we use $a_{L, B}$ as a factor that controls the magnitude of the embedded watermark. As in [Podilchuk and Zeng, 97] $a_{L, B}$ is also used as the threshold 
Table 1 Quantizers $Q_{L, B}$ for a four-level DWT with biorthogonal 9/7 filter

\begin{tabular}{|c|c|c|c|c|}
\hline \multirow{2}{*}{ Band } & \multicolumn{4}{|c|}{ Level } \\
\cline { 2 - 5 } & 1 & 2 & 3 & 4 \\
\hline HL,LH & 23.028 & 14.685 & 12.207 & 14.156 \\
\hline HH & 58.756 & 28.408 & 19.54 & 17.864 \\
\hline
\end{tabular}

that a wavelet coefficient is compared with in order to determine whether this coefficient is going to be watermarked or not. Consequently, the embedding equation is

$$
X_{L, B, i, j}^{\prime}= \begin{cases}X_{L, B, i, j}+b_{m} a_{L, B} w_{L, B, i, j}, & \text { if } X_{L, B, i, j}>a_{L, B} \\ X_{L, B, i, j}, & \text { otherwise }\end{cases}
$$

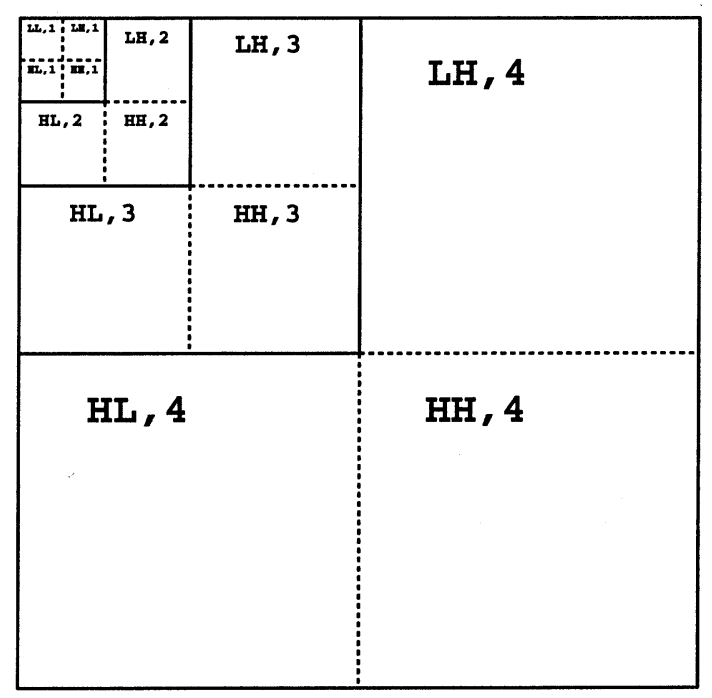

Figure 2 Subband naming conventions.

where $X_{L, B, i, j}$ refers to the wavelet coefficient at position $(i, j)$ of level $L$ and band $B, X_{L, B, i, j}^{\prime}$ refers to the watermarked wavelet coefficient and $w_{L, B, i, j}$ refers to the watermark coefficient (gaussian distributed) which is multiplied by the bit value $b_{m}(1$ or -1$)$ that is embedded in each wavelet coefficient. $K$ bits are embedded in each image. In each band of the first level the index $m$ is different for each wavelet coefficient. 
Therefore a different bit is embedded in each wavelet coefficient. If the number of the bits to be be embedded is smaller than the number of the wavelet coefficients of the level, the bit sequence is repeated until the wavelet coefficients run out. In the next level, the area of wavelet coefficients into which each bit $b_{m}$ is embedded is doubled in both the horizontal and vertical direction. Therefore, the number of coefficients in which each bit is embedded in a level, is four times the number of coefficients marked in the coarser level. The bit allocation procedure is schematically described in Figure 3.

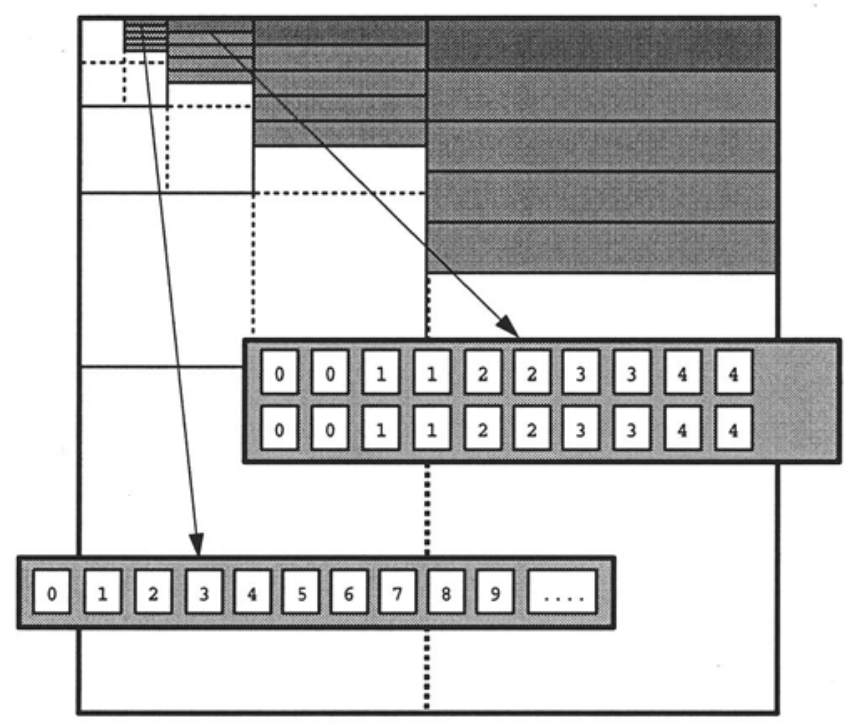

Figure 3 Repeated placement of watermark bits in the wavelet domain.

The maximum number of bits that can be imperceptibly embedded in an image depends on its size, content, as well as the possible attacks (cropping, filtering, compression, etc) that it may undergo. In order to increase the number of embedded bits and/or improve the robustness against possible attacks, error correcting codes may be used [Lin and Costello, 1982]. Error correcting codes can detect and correct errors that may occur in the embedded bitstream. In order to be able to correct possible errors, error control bits should be added in the embedded bitstream. Therefore, out of the $K$ bits that are embedded in the image, only $N$ constitute useful information bits. For this paper, a simple Hamming code is used that adds three error control bits $C_{1}, C_{2}, C_{3}$ for every four information bits $I_{1}, I_{2}, I_{3}, I_{4}$. The control bits are computed from the information bits in the following way 


$$
\begin{aligned}
& C_{1}=I_{1} \oplus I_{2} \oplus I_{4} \\
& C_{2}=I_{1} \oplus I_{3} \oplus I_{4} \\
& C_{3}=I_{2} \oplus I_{3} \oplus I_{4}
\end{aligned}
$$

Thus, the embedding bitstream takes the form $C_{1}, C_{2}, I_{1}, C_{3}, I_{2}, I_{3}, I_{4}$ for every four information bits. If only a single error occurs while recovering the four information bits, the error can be traced and corrected.

\section{WATERMARK RETRIEVAL AND ERROR CORRECTION}

The detection of the embedded bits is performed without the use of the original image. Similar to the embedding process, the wavelet coefficients $Y_{L, B, i, j}$ that are assumed to have been watermarked are determined by comparing $Y_{L, B, i, j}$ with $a_{L, B}$. If $Y_{L, B, i, j}>a_{L, B}$ then the watermarked, and also possibly distorted by compression or attacks, wavelet coefficient $Y_{L, B, i, j}$ is multiplied with the watermark $w_{L, B, i, j}$, which is derived from the meaningful message that the owner has used in the embedding process

$$
Z_{L, B, i, j}=Y_{L, B, i, j} w_{L, B, i, j}, \text { if } Y_{L, B, i, j}>a_{L, B}
$$

It should be noted, that some coefficients $X_{L, B, i, j}$ that exceeded the threshold $a_{L, B}$ and were watermarked as in equation (1) with a negative factor $b_{m} a_{L, B} w_{L, B, i, j}$ may obtain a value $Y_{L, B, i, j}$ below the threshold $a_{L, B}$ and therefore may not be used in the watermark retrieval process. The number of these coefficients was found to be very small and no significant deterioration in the performance of the watermark retrieval was observed. Therefore, no special care for such coefficients was taken.

Then, the set of all the $Z_{L, B, i, j}$ that were calculated is divided in $K$ subsets, each one corresponding to one of the $K$ bits of the embedded bitstream (see Figure 3). For each one of the $K$ subsets the bit value $b_{m}, m=1, \ldots, K$ is calculated as follows

$$
b_{m}=\operatorname{sign}\left(\frac{1}{N_{m}} \sum_{i=1}^{N_{m}} Z_{m}(i)\right)
$$

where $\left\{Z_{m}\right\}$ is the $m$ th subsequence of $\left\{Z_{L, B, i, j}\right\}$ that corresponds to $b_{m}$ and $N_{m}$ is the number of elements of $\left\{Z_{m}\right\}$. After the $K$ bits of the embedded bitstream are extracted, error correction is applied. For each sequence of seven bits $C_{1}, C_{2}, I_{1}, C_{3}, I_{2}, I_{3}, I_{4}$ the control bits $C_{1}^{\prime}, C_{2}^{\prime}, C_{3}^{\prime}$ 
are calculated from the information bits $I_{1}, I_{2}, I_{3}, I_{4}$ as in the embedding process. If $C_{1}^{\prime}, C_{2}^{\prime}, C_{3}^{\prime}$ are the same as $C_{1}, C_{2}, C_{3}$ then no correction of the information bits is needed. If error correction is needed, the bitstrings $C_{3} C_{2} C_{1}$ and $C_{3}^{\prime} C_{2}^{\prime} C_{1}^{\prime}$ are added using binary addition. The resulting sum indicates the position of the incorrectly extracted bit and its value is flipped. In this way, all $N$ corrected information bits are obtained.

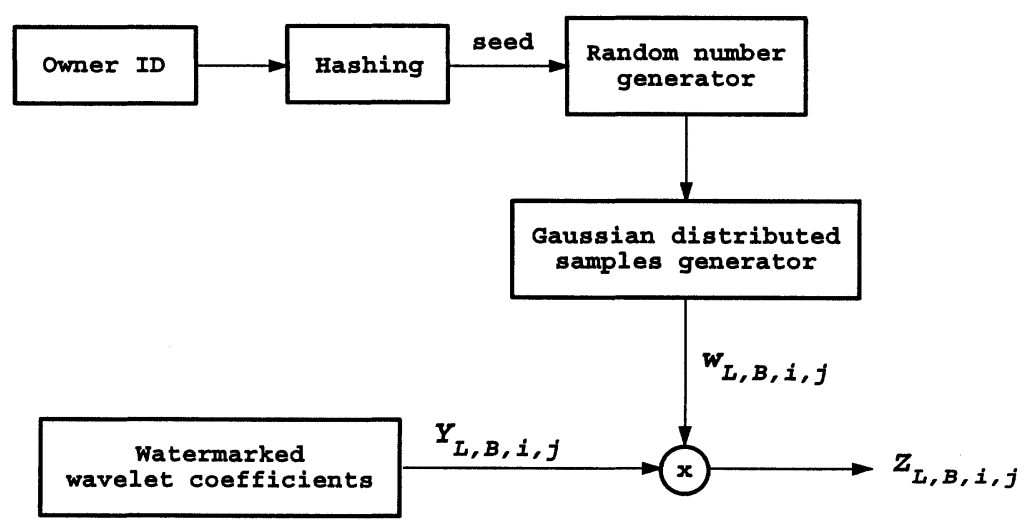

Figure 4 Detection scheme.

\section{EXPERIMENTAL RESULTS}

A software simulation of the proposed algorithm was implemented. The implementation is incorporated in the JPEG2000 verification model (VM 7.2). The watermark bits are immediately embedded after the image is wavelet transformed. Detection of the watermark bits is performed in the decoder before the inverse wavelet transform is performed. The JPEG2000 decoder supports progressive decoding [Taubman, 2000]. Therefore, a JPEG2000 compressed image may be partially decoded in order to reach a specific quality. The watermark detection is compatible with this procedure since it is able to extract the embedded bits from the set of the watermarked coefficients so far decoded.

For testing the detector performance we embedded bitstreams having length from $N=36$ to $N=108$ information bits or for $K=63$ to $K=189$ embedded bits. Figure 5 illustrates the detector performance for various lengths of the embedded bitstream and various decoding bitrates for the test image "Lena". As the bitrate increases and/or as the embedded bitstream length decreases, the detection performance improves. The detector performance is measured as the ratio of the number of the successfully decoded information bits over the total num- 
ber of bits. It is worth noting that if the decoding bitrate exceeds 0.5 bits/pixel for nearly all the tested embedded bitstream lengths all bits can be correctly extracted. In figures 6 and 7 the original and the watermarked "Lena" and "Barbara" images are shown respectively.

Finally cropping, blur filtering and JPEG compression (quality factor 60) attacks have been applied in the watermarked image "Barbara". 36 information bits were embedded in the original image and were all successfully retrieved from the attacked images (see Figure 8).

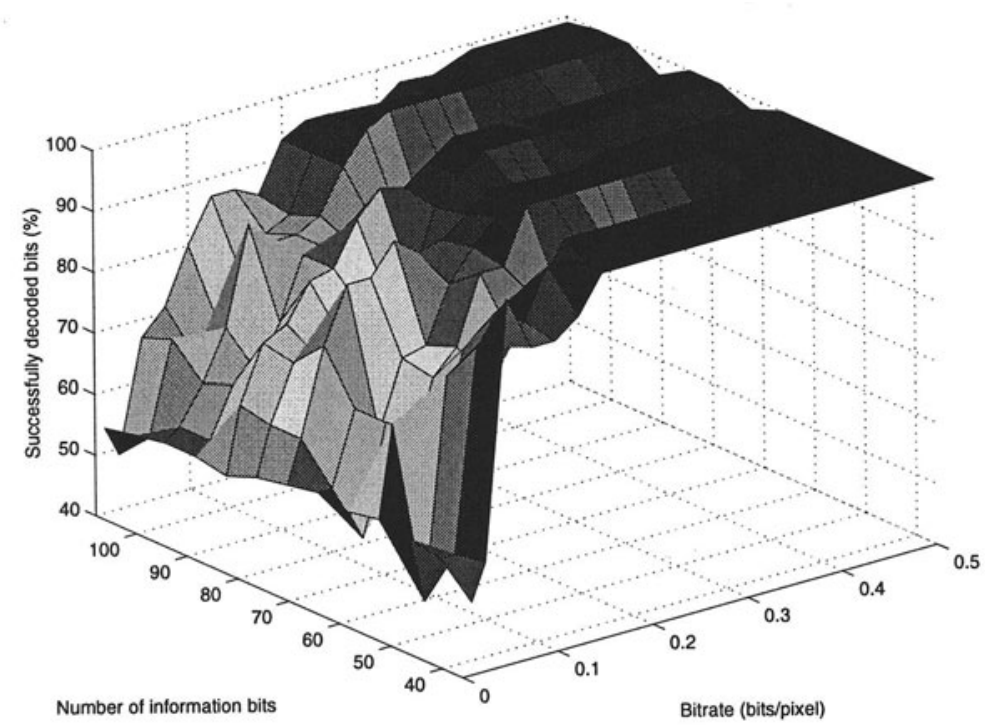

Figure 5 "Lena" multiple bit detection.

\section{CONCLUSION}

In this paper, a novel watermarking technique for embedding information in JPEG2000 images was presented. The proposed scheme embeds the watermark in wavelet coefficients and is compatible with the line-based discrete wavelet transform that is used by JPEG2000. The proposed method was coupled with wavelet-domain perceptual analysis to ensure that the watermark is invisible. The information bits carried by the watermark signal were protected using error correction codes. Watermark detection and information extraction were performed without the need of the original image. Experimental evaluation showed the 


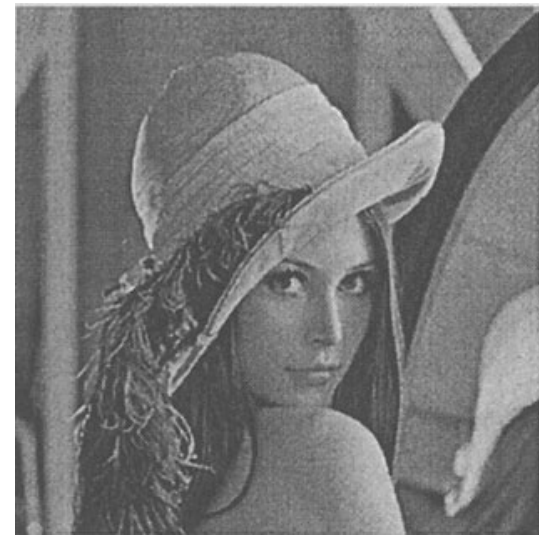

(a)

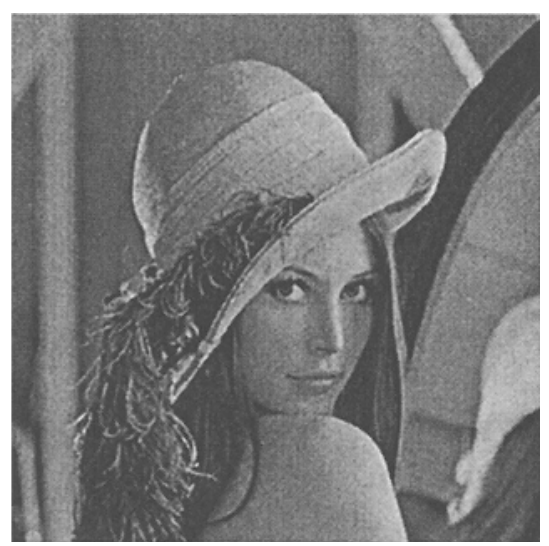

(b)

Figure 6 "Lena" (a) Original, (b) Watermarked.

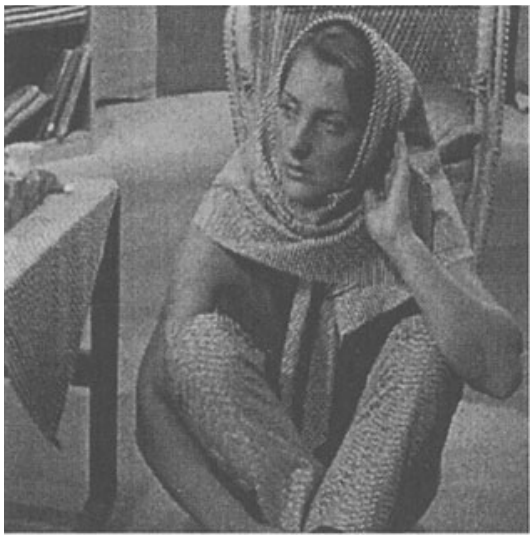

(a)

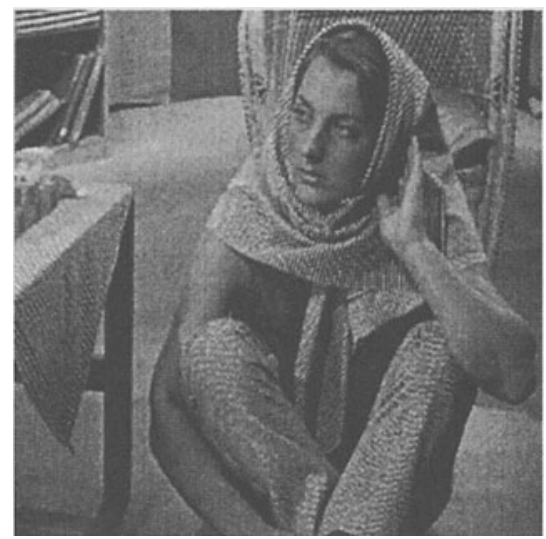

(b)

Figure 7 "Barbara" (a) Original, (b) Watermarked.

reliability of the proposed watermarking method when used with the recently finalized JPEG2000 standard.

\section{Acknowledgments}

This work was supported by the EC IST Project "ASPIS" and the Greek GSRT Project "PANORAMA". 


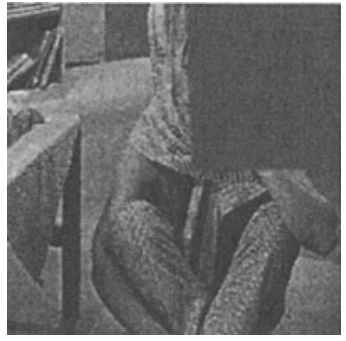

(a)

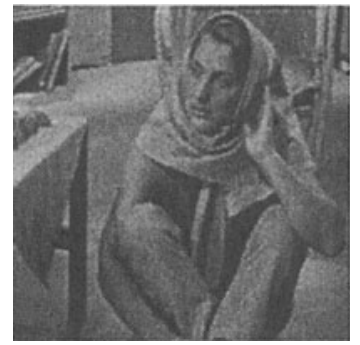

(b)

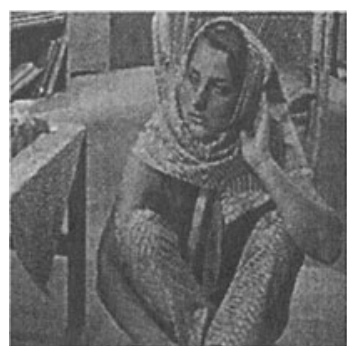

(c)

Figure 8 Attacks performed on image "Barbara" (a) Cropping, (b) Blur filtering, (c) JPEG compression (quality factor 60).

\section{References}

Antonini, M., Barlaud, M., Mathieu, P., and Daubechies, I. (1992). Image Coding Using Wavelet Transform. IEEE Trans. Image Processing, 1(2):205-210.

Lin, S. and Costello, D. J. (1982). Error Control Coding: Fundamentals and Applications. Prentice-Hall.

Podilchuk, C. I. and Zeng, W. (97). Perceptual watermarking of still images. In Proc. IEEE Workshop Multimedia Signal Processing, Princeton, NJ.

Su, P.-C., Wang, H.-J., and Kuo, C.-C. J. (2000). An Integrated Approach to Image Watermarking and JPEG2000 Compression. Journal of VLSI Signal Processing.

Taubman, D. (2000). High Performance Scalable Image Compression with EBCOT. IEEE Trans. Image Processing, 9(7):1158-1170.

Watson, A. B., Yang, G. Y., Solomon, J. A., and Villasenor, J. (1996). Visual thresholds for wavelet quantization error. In Proc. SPIE Conf. Human Vision and Electronic Imaging, volume 2657, pages 381-392. 